\title{
Strongly Gorenstein-projective Quiver Representations
}

\author{
Tengxia Ju and Xiu-Hua Luo*
}

\begin{abstract}
Given a field $k$, a finite-dimensional $k$-algebra $A$, and a finite acyclic quiver $Q$, let $A Q$ be the path algebra of $Q$ over $A$. Then the category of representations of $Q$ over $A$ is equivalent to the category of $A Q$-modules. The main result of this paper explicitly describes the strongly Gorenstein-projective $A Q$-modules via the separated monic representations with a local strongly Gorenstein-property. As an application, a necessary and sufficient condition is given on when each Gorenstein-projective $A Q$ module is strongly Gorenstein-projective. As a direct result, for an integer $t \geq 2$, let $A=k[x] /\left\langle x^{t}\right\rangle$, each Gorenstein-projective $A Q$-module is strongly Gorensteinprojective if and only if $A=k[x] /\left\langle x^{2}\right\rangle$.
\end{abstract}

\section{Introduction}

In 1969, Auslander and Bridger [2] introduced the finitely generated modules of G-dimension zero over Noetherian rings. In 1995, Enochs and Jenda [12] introduced Gorensteinprojective modules not necessarily finitely generated and over not necessarily Noetherian rings, via complete projective resolutions, and it turned out that Gorenstein-projective modules coincide with modules of G-dimension zero. Here let $A$ be an artin algebra, and $A$-mod be the category of finitely generated left $A$-modules. A complete A-projective resolution is an exact sequence of finitely generated projective $A$-modules

$$
P^{\bullet}=\cdots \longrightarrow P^{-1} \longrightarrow P^{0} \stackrel{d^{0}}{\longrightarrow} P^{1} \longrightarrow \cdots
$$

such that $\operatorname{Hom}_{A}\left(P^{\bullet}, A\right)$ is also exact. A module $M \in A$-mod is Gorenstein-projective [12], if there exists a complete $A$-projective resolution $P^{\bullet}$ such that $M \cong \operatorname{Ker} d^{0}$. Moreover, if all the modules and morphisms in $P^{\bullet}$ are the same, then $M$ is called a strongly Gorensteinprojective $A$-module $[7]$. Let $\mathcal{P}(A)$ (resp. $\mathcal{G P}(A), \mathcal{S G P}(A)$ ) be the full subcategory of $A$-mod of projective (resp. Gorenstein-projective, strongly Gorenstein-projective) modules. Then $\mathcal{P}(A) \subseteq \mathcal{S} \mathcal{G} \mathcal{P}(A) \subseteq \mathcal{G P}(A) \subseteq{ }^{\perp} A=\left\{X \in A\right.$-mod $\left.\mid \operatorname{Ext}_{A}^{i}(X, A)=0, \forall i \geq 1\right\}$.

Received August 3, 2020; Accepted November 19, 2020.

Communicated by Kunio Yamagata.

2020 Mathematics Subject Classification. 16G10, 16E65.

Key words and phrases. quiver representations, separated monic representations, (strongly) Gorensteinprojective modules, upper triangular matrix algebra.

Luo is supported by the National Science Foundation of China (No. 11771272).

*Corresponding author. 
It is easy to see that generally $\mathcal{S G P}(A) \neq \mathcal{G} \mathcal{P}(A)$. Mahdou and Tamekkante [21] studied when every Gorenstein-projective module is strongly Gorenstein-projective. The class $\mathcal{G P}(A)$ has been applied in many fields such as relative homological algebra 11, 12, algebra representation theory $[3,6,13,16$ and the Tate cohomology of algebra [5]. Holm [14] proved that $\mathcal{G P}(A)$ is also a projectively resolving subcategory of $A$-mod. In general, $\mathcal{S G P}(A)$ may not be a resolving subcategory of $A$-mod [24], however, it's still closed under extensions which is implied by Proposition 2.9 of $[7]$.

The construction of Gorenstein-projective modules has been extensively studied by many researchers such as Zhang, W. Li, F. Li, Ye, Hu, Xiong, and Zhou 15, 17, 20, 25, 26]. In 15, 19, 20, the authors described the Gorenstein-projective modules via separated monic representations. In this paper, the relation between strongly Gorenstein-projective modules and separated monic representations will be given. In Section 2, we investigate some properties of separated monic quiver representations. In Section 3 , we give an explicit description of strongly Gorenstein-projective modules of a finite acyclic quiver $Q$ over a finite-dimensional $k$-algebra $A$ where $k$ is a field. This is the main result of this paper. In Section 4, we give some applications of the main result.

\section{Properties of separated monic quiver representations}

Given a quiver $Q=\left(Q_{0}, Q_{1}, s, e\right)$ and an algebra $A$, let $\Lambda=A Q$ be the path algebra of $Q$ over $A$. A representation of $Q$ over $A$ is a data $X=\left(X_{i}, X_{\alpha}, i \in Q_{0}, \alpha \in Q_{1}\right)$ where $X_{i}$ is an $A$-module assigned to the vertex $i$ and $X_{\alpha}$ is an $A$-map assigned to the arrow $\alpha$. Then the category $\operatorname{rep}(Q, A)$ consisting of all representations of $Q$ over $A$ is equivalent to $A Q$-mod the category of modules over the path algebra $A Q$. The proof of this assertion is similar to the case when $A=k$ (see [4, Theorem 1.5, p .57] or [22, p. 44]). For the details and notations, one can refer to $[19$.

For vertex $i, j \in Q_{0}$, let $\mathcal{A}(j \rightarrow i)$ be the set of all arrows from $j$ to $i, \mathcal{A}(\cdot \rightarrow i)$ be the set of all arrows ending in $i$, and $\mathcal{A}(j \rightarrow \cdot)$ be the set of all arrows starting in $j$. Let $\mathcal{P}(j \rightarrow i)$ denote the set of all paths with length no less than 1 starting in $j$ and ending in $i$ and $|\mathcal{P}(j \rightarrow i)|$ be the number of paths in $\mathcal{P}(j \rightarrow i)$. For each vertex $i \in Q_{0}$, there exists an evaluation functor $-_{i}: \operatorname{rep}(Q, A) \rightarrow A$-mod sending each representation $X$ to the $i$-th branch $X_{i}$. This functor has been studied by many researchers $[1,9,10,19]$. Define $I_{i}(\cdot): A-\bmod \rightarrow \operatorname{rep}(Q, A)$ as follows: for each $M \in A-\bmod$, let $I_{i}(M)_{j}=M^{|p(j \rightarrow i)|}$ be the direct sum of $|\mathcal{P}(j \rightarrow i)|$ copies of $M$, and the maps $I_{i}(M)_{\alpha}$ be natural projections. Then $\left(-{ }_{i}, I_{i}(\cdot)\right)$ is an adjoint pair. The left adjoint $P_{i}(\cdot)$ of $-{ }_{i}$ is defined dually: for each $M \in A$-mod, one defines $P_{i}(M)_{j}=M^{|\mathcal{P}(i \rightarrow j)|}$ and the maps $P_{i}(M)_{\alpha}$ are natural injections. For each injective $A$-module $J, I_{i}(J)$ is an injective object in $\operatorname{rep}(Q, A)$, and for each projective $A$-module $P, P_{i}(P)$ is a projective object in $\operatorname{rep}(Q, A)$. 
In order to describe the strongly Gorenstein-projective $\Lambda$-modules, we need the following key definition which was introduced in [19].

Definition 2.1. A representation $X=\left(X_{i}, X_{\alpha}, i \in Q_{0}, \alpha \in Q_{1}\right)$ of $Q$ over $A$ is a monic representation, or a monic $A Q$-module, if the following condition is satisfied

(m1) For each $\alpha \in Q_{1}, X_{\alpha}: X_{s(\alpha)} \rightarrow X_{e(\alpha)}$ is an injective morphism.

Moreover, if a monic representation satisfies the condition:

(m2) For each $i \in Q_{0}$, there holds $\sum_{\alpha \in \mathcal{A}(\cdot \rightarrow i)} \operatorname{Im} X_{\alpha}=\bigoplus_{\alpha \in \mathcal{A}(\cdot \rightarrow i)} \operatorname{Im} X_{\alpha}$, then it is called a separated monic representation.

We denote by $\operatorname{mon}(Q, A)$ the category of monic representations, and $\operatorname{smon}(Q, A)$ the category of separated monic representations.

Remark 2.2. (1) One can see that the conditions (m1) and (m2) are equivalent to the condition: $\left(X_{\alpha}\right)_{\alpha \in \mathcal{A}(\cdot \rightarrow i)}: \bigoplus_{\alpha \in \mathcal{A}(\cdot \rightarrow i)} X_{s(\alpha)} \rightarrow X_{i}$ is a monomorphism, for each vertex $i \in Q_{0}$.

(2) By the definitions of separated monic representations and the functor $P_{i}(\cdot)$, we know that $P_{i}(M)$ is a separated monic representation of $Q$ over $A$ for each $A$-module $M$ and for each vertex $i \in Q_{0}$. For each strongly Gorenstein-projective $A$-module $M$ with a complete projective resolution $P^{\bullet}, P_{i}(M)$ is a strongly Gorenstein-projective $A Q$-module with a complete projective resolution $P_{i}\left(P^{\bullet}\right)$.

In the following, given a finite acyclic quiver $Q=\left(Q_{0}, Q_{1}, s, e\right), Q_{0}$ is labeled as $1, \ldots, n$ such that $s(\alpha)>e(\alpha)$ for each $\alpha \in Q_{1}$, then $n$ is a source and 1 is a sink. We also write a representation $X$ of $Q$ over $A$ as $\left(\begin{array}{c}X_{1} \\ \vdots \\ X_{n}\end{array}\right)_{\left(X_{\alpha}, \alpha \in Q_{1}\right)}$, and a morphism in $\operatorname{rep}(Q, A)$ can be written as $\left(\begin{array}{c}f_{1} \\ \vdots \\ f_{n}\end{array}\right)$. Let $Q^{\prime}$ be the quiver obtained from $Q$ by deleting the source $n$, $P=\bigoplus_{\alpha \in \mathcal{A}(n \rightarrow \cdot)} P_{e(\alpha)}(A)$, then $A Q=\left(\begin{array}{cc}A Q^{\prime} & P \\ 0 & A\end{array}\right)$ and a representation of $Q$ over $A$ can be written as $X=\left(\begin{array}{c}X^{\prime} \\ X_{n}\end{array}\right)_{\phi}$ where $X^{\prime} \in A Q^{\prime}-\bmod$ and $\phi=\left(\begin{array}{c}\phi_{1} \\ \phi_{2} \\ \vdots \\ \phi_{n-1}\end{array}\right): P \otimes_{A} X_{n} \rightarrow X^{\prime}$ with $\phi_{i}=\left(X_{p_{1}}, X_{p_{2}}, \ldots, X_{p_{m_{i}}}\right)$ where $\left\{p_{1}, p_{2}, \ldots, p_{m_{i}}\right\}=\mathcal{P}(n \rightarrow i)$ is the set of nontrivial paths from $n$ to $i$.

In [19], the properties of separated monic representations have been studied extensively. Here, we will list two properties which were proved in [19] and give a new property. 
Proposition 2.3. 19 Let $X=\left(\begin{array}{c}X^{\prime} \\ X_{n}\end{array}\right)_{\phi}$ be a separated monic $\Lambda$-module. Then

(1) For each $i \in Q_{0}^{\prime}$, there holds $\sum_{p \in \mathcal{P}(n \rightarrow i)} \operatorname{Im} X_{p}=\bigoplus_{p \in \mathcal{P}(n \rightarrow i)} \operatorname{Im} X_{p}$;

(2) $\phi: P \otimes_{A} X_{n} \rightarrow X^{\prime}$ is injective;

(3) $\operatorname{Cok} \phi=\left(X_{i} / \bigoplus_{p \in \mathcal{P}(n \rightarrow i)} \operatorname{Im} X_{p}, \widetilde{X_{\alpha}}, i \in Q_{0}^{\prime}, \alpha \in Q_{1}^{\prime}\right)$ where for each $\alpha: j \rightarrow i$ in $Q_{1}^{\prime}$,

$$
\widetilde{X_{\alpha}}: X_{j} / \bigoplus_{q \in \mathcal{P}(n \rightarrow j)} \operatorname{Im} X_{q} \rightarrow X_{i} / \bigoplus_{p \in \mathcal{P}(n \rightarrow i)} \operatorname{Im} X_{p}
$$

is the A-map induced by $X_{\alpha}$.

Proposition 2.4. 19 Let $X=\left(\begin{array}{c}X^{\prime} \\ X_{n}\end{array}\right)_{\phi}$ be a separated monic $\Lambda$-module. Then Cok $\phi$ is a separated monic $\Lambda^{\prime}$-module.

In [19, the authors used separated monic representations and the local Gorensteinprojective condition to describe Gorenstein-projective $A Q$-modules. To describe strongly Gorenstein-projective $A Q$-modules, we need to introduce the local strongly Gorensteinprojective condition. Given a quiver $Q$, for a representation $X=\left(X_{i}, X_{\alpha}\right)$, if for each vertex $i \in Q_{0}, X_{i}$ and $\operatorname{Cok}_{i}(X):=X_{i} / \sum_{\alpha \in \mathcal{A}(\rightarrow i)} \operatorname{Im} X_{\alpha}$ are Gorenstein-projective (resp. strongly Gorenstein-projective) modules, then we say that $X=\left(X_{i}, X_{\alpha}\right)$ satisfies the local Gorenstein-projective (resp. strongly Gorenstein-projective) condition, or the condition $(G)$ (resp. $(S G))$ for short.

Proposition 2.5. Let $X=\left(\begin{array}{c}X^{\prime} \\ X_{n}\end{array}\right)_{\phi}$ be a separated monic $\Lambda$-module satisfying the condition $(S G)$. Then Cok $\phi$ also satisfies the condition $(S G)$.

Proof. In order to prove this assertion, firstly, we need to prove that the strongly Gorensteinprojective modules are closed under extensions. That is, for an exact sequence $0 \rightarrow X \rightarrow$ $Y \rightarrow Z \rightarrow 0$ with $X, Z$ being strongly Gorenstein-projective modules, then so is $Y$. In fact, since $X, Z$ are Gorenstein-projective modules, we have $\operatorname{Ext}^{i}(Y, P)=0$ for $i \geq 1$ and for each projective module $P$. By Proposition 2.9 of [7], we just need to prove that there is an exact sequence $0 \rightarrow Y \rightarrow P^{\prime} \rightarrow Y \rightarrow 0$ with $P^{\prime}$ being projective. Since $X, Z$ are strongly Gorenstein-projective modules, there are two exact sequences $0 \rightarrow X \rightarrow P_{1} \rightarrow X \rightarrow 0$ and $0 \rightarrow Z \rightarrow P_{2} \rightarrow Z \rightarrow 0$ with $P_{1}, P_{2}$ being projective. Since projective modules are relative injective objects in the category of Gorenstein-projective modules, we have a commutative diagram 


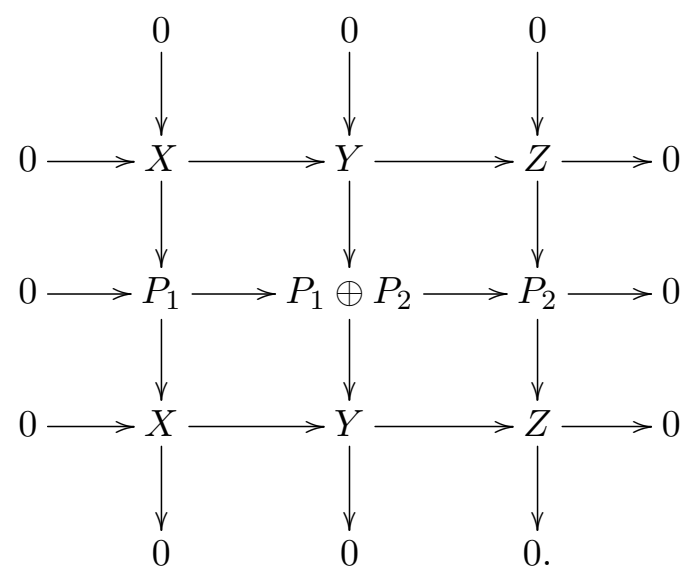

Hence $0 \rightarrow Y \rightarrow P_{1} \oplus P_{2} \rightarrow Y \rightarrow 0$ is exact. So $Y$ is a strongly Gorenstein-projective module.

By Proposition 2.3, the $i$-th branch of $\operatorname{Cok} \phi$ is $X_{i} / \bigoplus_{p \in \mathcal{P}(n \rightarrow i)} \operatorname{Im} X_{p}$ and the strongly Gorenstein-projective modules are closed under extensions. Then the assertion that $X_{i} / \bigoplus_{p \in \mathcal{P}(n \rightarrow i)} \operatorname{Im} X_{p}$ is strongly Gorenstein-projective can be proved similarly to 19 , Lemma 5.8].

By the proof of Lemma 5.7 of [19, we know that

$$
\begin{aligned}
\operatorname{Cok}_{i}(\operatorname{Cok} \phi) & =\left(X_{i} / \bigoplus_{p \in \mathcal{P}(n \rightarrow i)} \operatorname{Im} X_{p}\right) / \bigoplus_{\substack{\alpha \in Q_{1}^{\prime} \\
e(\alpha)=i}} \operatorname{Im} \widetilde{X_{\alpha}} \\
& \cong X_{i} / \bigoplus_{\beta \in \mathcal{A}(\cdot \rightarrow i)} \operatorname{Im} X_{\beta}=\operatorname{Cok}_{i}(X)
\end{aligned}
$$

is strongly Gorenstein-projective since $X$ satisfies the condition $(S G)$. Hence Cok $\phi$ satisfies the condition $(S G)$.

\section{Strongly Gorenstein-projective modules}

Let $A$ and $B$ be rings, $M$ an $A$-B-bimodule, and $\Lambda=\left(\begin{array}{cc}A & M \\ 0 & B\end{array}\right)$ the upper triangular matrix ring where the addition and the multiplication are given by the ones of matrices. We assume that $\Lambda$ is an artin algebra [4, p. 72], and consider finitely generated $\Lambda$-modules. A $\Lambda$-module can be identified with a triple $\left(\begin{array}{c}X \\ Y\end{array}\right)_{\phi}$, or simply $\left(\begin{array}{l}X \\ Y\end{array}\right)$ if $\phi$ is clear, where $X \in$ $A$-mod, $Y \in B$-mod, and $\phi: M \otimes_{B} Y \rightarrow X$ is an $A$-map. The indecomposable projective $\Lambda$-modules are of the form $\left(\begin{array}{c}P \\ 0\end{array}\right),\left(\begin{array}{c}M \otimes_{B} Q \\ Q\end{array}\right)_{\text {id }}$, where $P$ is an indecomposable projective $A$-module and $Q$ is an indecomposable projective $B$-module. A $\Lambda$-map $\left(\begin{array}{l}X \\ Y\end{array}\right)_{\phi} \rightarrow\left(\begin{array}{l}X^{\prime} \\ Y^{\prime}\end{array}\right)_{\phi^{\prime}}$ can be identified with a pair $\left(\begin{array}{l}f \\ g\end{array}\right)$, where $f \in \operatorname{Hom}_{A}\left(X, X^{\prime}\right), g \in \operatorname{Hom}_{B}\left(Y, Y^{\prime}\right)$, such that $f \phi=\phi^{\prime}\left(\operatorname{id}_{M} \otimes g\right)$. 
In [19], the authors described the construction of Gorenstein-projective modules over upper triangular matrix rings when ${ }_{A} M$ and $M_{B}$ are projective modules. In [26], Zhang extended the result under a much weaker condition, namely, when ${ }_{A} M_{B}$ is a compatible $A$ $B$-bimodule. Here, we can get a similar result on strongly Gorenstein-projective modules when ${ }_{A} M$ and $M_{B}$ are projective modules.

Theorem 3.1. Let $\Lambda=\left(\begin{array}{cc}A & M \\ 0 & B\end{array}\right)$ be an artin algebra, $M$ an $A$-B-bimodule such that ${ }_{A} M$ and $M_{B}$ are projective modules. Then $(\underset{Y}{X})_{\phi} \in \mathcal{S G \mathcal { P }}(\Lambda)$ if and only if $\phi: M \otimes_{B} Y \rightarrow X$ is an injective $A$-map, $\operatorname{Cok} \phi \in \mathcal{S G P}(A)$, and $Y \in \mathcal{S G P}(B)$.

Proof. Firstly, we prove the sufficiency. Assume that $\phi: M \otimes_{B} Y \rightarrow X$ is injective, $\operatorname{Cok} \phi \in \mathcal{S G P}(A)$, and $Y \in \mathcal{S G P}(B)$. Then we have a complete $B$-projective resolution

$$
Q^{\bullet}=\cdots \longrightarrow Q \stackrel{d^{\prime}}{\longrightarrow} Q \stackrel{d^{\prime}}{\longrightarrow} Q \longrightarrow \cdots
$$

with $Y=\operatorname{Ker} d^{\prime}$, and a complete $A$-projective resolution

$$
P^{\bullet}=\cdots \longrightarrow P \stackrel{d}{\longrightarrow} P \stackrel{d}{\longrightarrow} P \longrightarrow \cdots
$$

with $\operatorname{Cok} \phi=\operatorname{Ker} d$. Let $d^{\prime}$ be the composition $Q \stackrel{e^{\prime}}{\longrightarrow} Y \stackrel{\sigma^{\prime}}{\longrightarrow} Q$ and $d$ be the composition $P \stackrel{e}{\longrightarrow}$ Cok $\phi \stackrel{\sigma}{\longrightarrow} P$ where $e^{\prime}, e$ are epimorphisms, and $\sigma^{\prime}, \sigma$ are injective morphisms. Since $M_{B}$ is projective, we get the following exact sequences of $A$-modules

$$
\begin{gathered}
0 \longrightarrow M \otimes_{B} Y \longrightarrow M \otimes_{B} Q \longrightarrow M \otimes_{B} Q \longrightarrow \cdots, \\
0 \longrightarrow \operatorname{Cok} \phi \longrightarrow P \longrightarrow P \longrightarrow
\end{gathered}
$$

Since ${ }_{A} M$ is projective, $M \otimes_{B} Q$ is a projective $A$-module. Since $\operatorname{Ext}_{A}^{1}\left(\operatorname{Cok} \phi, P^{\prime}\right)=0$ for any projective $A$-module $P^{\prime}$, it follows from the exact sequence $0 \rightarrow M \otimes_{B} Y \rightarrow X \rightarrow$ Cok $\phi \rightarrow 0$ that there is a commutative diagram

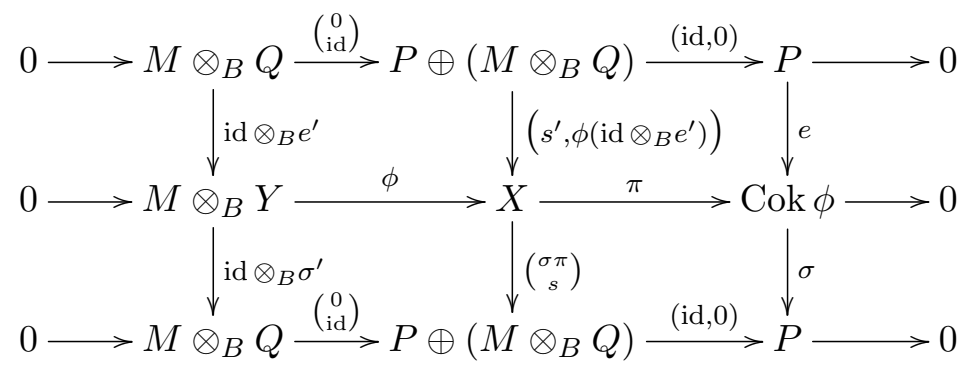

with exact rows and columns, and $s \phi\left(\mathrm{id} \otimes_{B} e^{\prime}\right)=\mathrm{id} \otimes_{B} d^{\prime}, \sigma \pi s^{\prime}=d$ where $\pi: X \rightarrow$ Cok $\phi$ is the canonical epimorphism induced by $\phi$. Hence we get an exact sequence of $A$-modules

$$
\cdots \longrightarrow P \oplus\left(M \otimes_{B} Q\right) \stackrel{\partial}{\longrightarrow} P \oplus\left(M \otimes_{B} Q\right) \stackrel{\partial}{\longrightarrow} P \oplus\left(M \otimes_{B} Q\right) \longrightarrow \cdots
$$


following from $P^{\bullet}$ and $M \otimes Q^{\bullet}$ being exact where $\partial=\left(\begin{array}{cc}d & 0 \\ s s^{\prime} & \text { id } \otimes_{B} d^{\prime}\end{array}\right), s s^{\prime}: P \rightarrow M \otimes_{B} Q$, such that the following sequence of projective $\Lambda$-modules

$$
L^{\bullet}=\cdots \longrightarrow\left(\begin{array}{c}
P \oplus\left(M \otimes_{B} Q\right) \\
Q
\end{array}\right)_{\left(\begin{array}{c}
0 \\
\mathrm{id}
\end{array}\right)}^{\stackrel{\left(\begin{array}{c}
\partial \\
d^{\prime}
\end{array}\right)}{\longrightarrow}}\left(\begin{array}{c}
P \oplus\left(M \otimes_{B} Q\right) \\
Q
\end{array}\right)_{\left(\begin{array}{c}
0 \\
\text { id }
\end{array}\right)}^{\stackrel{\left(\begin{array}{c}
\partial \\
d^{\prime}
\end{array}\right)}{\longrightarrow} \cdots}
$$

is exact with $\operatorname{Ker}\left(\begin{array}{c}\partial \\ d^{\prime}\end{array}\right)=\left(\begin{array}{c}X \\ Y\end{array}\right)_{\phi}$.

For each projective $A$-module $P^{\prime}, \operatorname{Hom}_{\Lambda}\left(L^{\bullet},\left(\begin{array}{c}P^{\prime} \\ 0\end{array}\right)\right) \cong \operatorname{Hom}_{A}\left(P^{\bullet}, P^{\prime}\right)$ is exact, since $P^{\bullet}$ is a complete projective resolution. For each projective $B$-module $Q^{\prime}$, since $Q^{\bullet}$ is a complete projective resolution, $\operatorname{Hom}_{B}\left(Q^{\bullet}, Q^{\prime}\right)$ is exact. Since $M \otimes_{B} Q^{\prime}$ is projective, $\operatorname{Hom}_{A}\left(P^{\bullet}, M \otimes_{B} Q^{\prime}\right)$ is exact. Note that

$$
\operatorname{Hom}_{\Lambda}\left(\left(\begin{array}{c}
P \oplus\left(M \otimes_{B} Q\right) \\
Q
\end{array}\right),\left(\begin{array}{c}
M \otimes_{B} Q^{\prime} \\
Q^{\prime}
\end{array}\right)\right) \cong \operatorname{Hom}_{A}\left(P, M \otimes_{B} Q^{\prime}\right) \oplus \operatorname{Hom}_{B}\left(Q, Q^{\prime}\right)
$$

By the canonical exact sequence of complexes

$$
0 \longrightarrow \operatorname{Hom}_{A}\left(P^{\bullet}, M \otimes_{B} Q^{\prime}\right) \stackrel{\left(\begin{array}{c}
\mathrm{id} \\
0
\end{array}\right)}{\longrightarrow} \operatorname{Hom}_{\Lambda}\left(L^{\bullet},\left(\begin{array}{c}
M \otimes_{B} Q^{\prime} \\
Q^{\prime}
\end{array}\right)\right) \stackrel{(0, \text { id })}{\longrightarrow} \operatorname{Hom}_{B}\left(Q^{\bullet}, Q^{\prime}\right) \longrightarrow 0
$$

we know that $\operatorname{Hom}_{\Lambda}\left(L^{\bullet},\left(\begin{array}{c}M \otimes_{B} Q^{\prime} \\ Q^{\prime}\end{array}\right)\right)$ is also exact. Therefore we conclude that $L^{\bullet}$ is a complete $\Lambda$-projective resolution, and hence $(\underset{Y}{X})_{\phi}$ is a strongly Gorenstein-projective $\Lambda$ module.

Conversely, assume that $(\underset{Y}{X})_{\phi} \in \mathcal{S G \mathcal { P }}(\Lambda)$. There is a complete $\Lambda$-projective resolution 3.2 with $\operatorname{Ker}\left(\begin{array}{c}2 \\ d^{\prime}\end{array}\right)=\left(\begin{array}{c}X \\ Y\end{array}\right)_{\phi}$. We can get an exact sequence 3.1 of projective $B$-modules with $\operatorname{Ker} d^{\prime}=Y$, and the following exact sequence

$$
V^{\bullet}=\cdots \longrightarrow P \oplus\left(M \otimes_{B} Q\right) \stackrel{\partial}{\longrightarrow} P \oplus\left(M \otimes_{B} Q\right) \stackrel{\partial}{\longrightarrow} P \oplus\left(M \otimes_{B} Q\right) \longrightarrow \cdots
$$

of projective $A$-modules with $\operatorname{Ker} \partial=X$. Since $M_{B}$ is projective, it follows that $M \otimes_{B} Q^{\bullet}$ is exact. Since $\left(\begin{array}{c}2 \\ d^{\prime}\end{array}\right)$ is a $\Lambda$-map, by 3.2 we know that $\partial$ is of the form $\partial=\left(\begin{array}{l}d \\ \eta \operatorname{id} \otimes_{B} d^{\prime}\end{array}\right)$, where $\eta: P \rightarrow M \otimes_{B} Q$, and

$$
P^{\bullet}=\cdots \longrightarrow P \stackrel{d}{\longrightarrow} P \stackrel{d}{\longrightarrow} P \longrightarrow \cdots
$$

is a complex. By the canonical exact sequence of complexes

$$
0 \longrightarrow M \otimes_{B} Q^{\bullet} \stackrel{\left(\begin{array}{l}
0 \\
\text { id }
\end{array}\right)}{\longrightarrow} V^{\bullet} \stackrel{(\mathrm{id}, 0)}{\longrightarrow} P^{\bullet} \longrightarrow 0
$$

we see that $P^{\bullet}$ is also exact. 
From 3.2 we have the following commutative diagram with exact rows and columns:

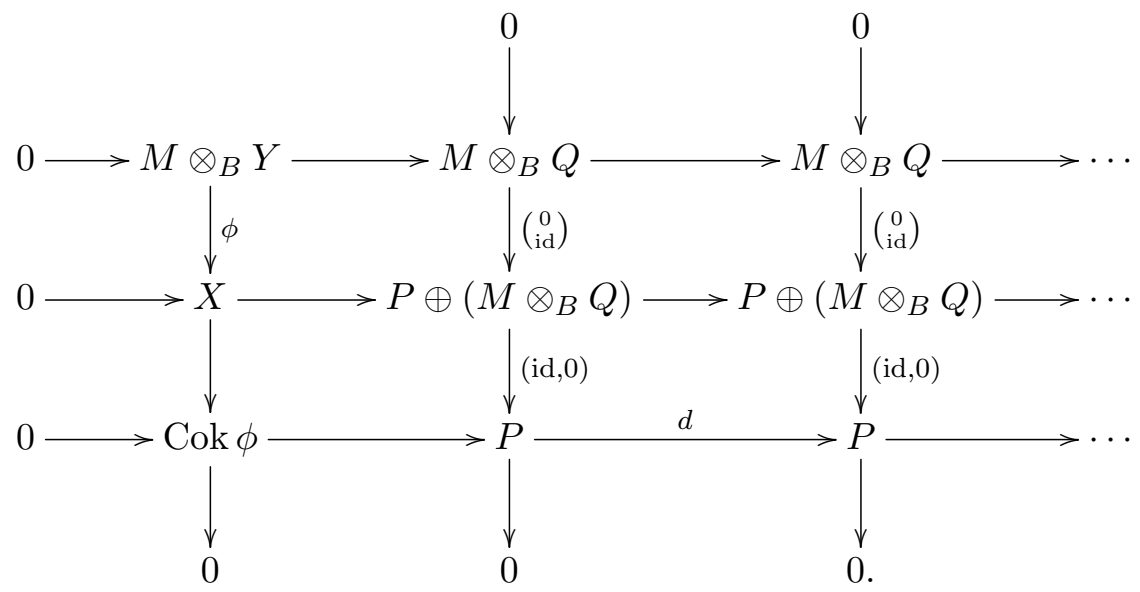

Thus $\phi: M \otimes_{B} Y \rightarrow X$ is injective and $\operatorname{Ker} d \cong \operatorname{Cok} \phi$. For each projective $A$-module $P^{\prime}$, since $\operatorname{Hom}_{\Lambda}\left(L^{\bullet},\left(\begin{array}{c}P^{\prime} \\ 0\end{array}\right)\right) \cong \operatorname{Hom}_{A}\left(P^{\bullet}, P^{\prime}\right)$ and $L^{\bullet}$ is a complete projective resolution, it follows that $P^{\bullet}$ is a complete projective resolution, and hence Cok $\phi$ is a strongly Gorensteinprojective $A$-module.

For each projective $B$-module $Q^{\prime}$, since $P^{\bullet}$ is a complete projective resolution, it follows that $\operatorname{Hom}_{A}\left(P^{\bullet}, M \otimes_{B} Q^{\prime}\right)$ is exact. Since $L^{\bullet}$ is a complete projective resolution, it follows that

$$
\operatorname{Hom}_{\Lambda}\left(L^{\bullet},\left(\begin{array}{c}
M \otimes_{B} Q^{\prime} \\
Q^{\prime}
\end{array}\right)\right) \cong \operatorname{Hom}_{A}\left(P^{\bullet}, M \otimes_{B} Q^{\prime}\right) \oplus \operatorname{Hom}_{B}\left(Q^{\bullet}, Q^{\prime}\right)
$$

is exact. Here the direct sum only means that each term of the complex at the left side is a direct sum of terms of complexes at the right side. It does not mean a direct sum of complexes. By the same argument we know that $\operatorname{Hom}_{B}\left(Q^{\bullet}, Q\right)$ is exact. It follows that $Y$ is a strongly Gorenstein-projective $B$-module. This completes the proof.

In [19], the authors gave an explicit description of Gorenstein-projective modules over the path algebra of a finite acyclic quiver $Q$ over an algebra $A$ via separated monic representations. The earlier similar result was given by Zhang in 25] when $Q$ is the quiver $A_{n}$ with linear orientation. Here, we give a relation between strongly Gorenstein-projective modules and separated monic representations.

Theorem 3.2. Let $Q$ be a finite acyclic quiver, $A$ a finite-dimensional algebra over a field k. Let $\Lambda=A Q$ and $X=\left(X_{i}, X_{\alpha}, i \in Q_{0}, \alpha \in Q_{1}\right)$ be a $\Lambda$-module. Then $X \in \mathcal{S G P}(\Lambda)$ if and only if $X \in \operatorname{smon}(Q, A)$ and $X$ satisfies the condition $(S G)$.

Theorem 3.2 will be proved by using Theorem 3.1 and induction on $\left|Q_{0}\right|$.

Remember we label $Q_{0}$ as $1, \ldots, n$, such that $j>i$ if $\alpha: j \rightarrow i$ is in $Q_{1}$. Thus $n$ is a source of $Q$. Denote by $Q^{\prime}$ the quiver obtained from $Q$ by deleting vertex $n$, and $\Lambda^{\prime}=A Q^{\prime}$. 
Put $P=\bigoplus_{\alpha \in \mathcal{A}(n \rightarrow \cdot)} P_{e(\alpha)}(A)$. Clearly $P$ is a $\Lambda^{\prime}-A$-bimodule and $\Lambda=\left(\begin{array}{cc}\Lambda^{\prime} & P \\ 0 & A\end{array}\right)$. Moreover, $P$ is a (left) projective $\Lambda^{\prime}$-module, and a (right) projective $A$-module. So we can apply Theorem 3.1. By a direct translation from Theorem 3.1] in this special case, we have

Lemma 3.3. Let $X=\left(\begin{array}{c}X^{\prime} \\ X_{n}\end{array}\right)_{\phi}$ be a $\Lambda$-module. Then $X \in \mathcal{S G P}(\Lambda)$ if and only if $X$ satisfies the following conditions:

(i) $X_{n} \in \mathcal{S} \mathcal{G} \mathcal{P}(A)$;

(ii) $\phi: P \otimes_{A} X_{n} \rightarrow X^{\prime}$ is injective;

(iii) $\operatorname{Cok} \phi \in \mathcal{S G P}\left(\Lambda^{\prime}\right)$.

Firstly, we prove the sufficiency of Theorem 3.2 . That is

Lemma 3.4. Let $Q$ be a finite acyclic quiver, $A$ a finite-dimensional algebra over a field $k$, and $\Lambda=A Q$. If $X=\left(X_{i}, X_{\alpha}, i \in Q_{0}, \alpha \in Q_{1}\right)$ is a separated monic $\Lambda$-module satisfying the condition $(S G)$, then $X$ is strongly Gorenstein-projective.

Proof. Using induction on $n=\left|Q_{0}\right|$. The assertion clearly holds for $n=1$. Suppose that the assertion holds for $\left|Q_{0}\right|=n-1$ with $n \geq 2$. For $\left|Q_{0}\right|=n$, it suffices to prove that $X$ satisfies the conditions (i), (ii) and (iii) in Lemma 3.3 .

The condition (i) is contained in $(S G)$; and the condition (ii) follows from Proposition 2.3 (2). By Proposition 2.4. Cok $\phi$ is a separated monic $\Lambda^{\prime}$-module; and by Proposition 2.5. we know that $\operatorname{Cok} \phi$ satisfies $(S G)$. It follows from the inductive hypothesis that the condition (iii) is satisfied.

Lemma 3.5. Let $X=\left(X_{i}, X_{\alpha}, i \in Q_{0}, \alpha \in Q_{1}\right)$ be a $\Lambda$-module with $X_{n}$ being a strongly Gorenstein-projective A-module. Then $P \otimes_{A} X_{n}$ is a strongly Gorenstein-projective $\Lambda^{\prime}$ module, where $P=\bigoplus_{\alpha \in \mathcal{A}(n \rightarrow \cdot)} P_{e(\alpha)}(A)$.

Proof. Since

$$
P \otimes_{A} X_{n}=\left(\bigoplus_{\alpha \in \mathcal{A}(n \rightarrow \cdot)} P_{e(\alpha)}(A)\right) \otimes_{A} X_{n} \cong \bigoplus_{\alpha \in \mathcal{A}(n \rightarrow \cdot)} P_{e(\alpha)}\left(X_{n}\right)
$$

then, by Remark 2.2, we can see that for each $\alpha \in \mathcal{A}(n \rightarrow \cdot), P_{e(\alpha)}\left(X_{n}\right)$ is a strongly Gorenstein-projective $\Lambda^{\prime}$-module. Now we complete the proof.

Secondly, we prove the necessity of Theorem 3.2 . That is

Lemma 3.6. If $X$ is a strongly Gorenstein-projective $\Lambda$-module, then $X$ is a separated monic $\Lambda$-module satisfying the condition $(S G)$. 
Proof. Since all strongly Gorenstein-projective modules are Gorenstein-projective, by Theorem 5.1 of $[19], X$ is a separated monic $\Lambda$-module.

Using induction on $n=\left|Q_{0}\right|$ to prove that $X$ satisfies the condition $(S G)$. The assertion is clear for $n=1$. Suppose that the assertion holds for $\left|Q_{0}\right|=n-1$ with $n \geq 2$, we need to prove that the assertion is true for $\left|Q_{0}\right|=n$. We write as $X=\left(\begin{array}{c}X^{\prime} \\ X_{n}\end{array}\right)_{\phi}$. Then $X$ satisfies the conditions (i), (ii) and (iii) in Lemma 3.3 .

By the condition (i) and Lemma 3.5, we know that $P \otimes_{A} X_{n}$ is a strongly Gorensteinprojective $\Lambda^{\prime}$-module. Then by the conditions (ii) and (iii), we know that $X^{\prime} \in \mathcal{S G P}\left(\Lambda^{\prime}\right)$ since $0 \rightarrow P \otimes_{A} X_{n} \rightarrow X^{\prime} \rightarrow \operatorname{Cok} \phi \rightarrow 0$ is exact and $\mathcal{S G \mathcal { P }}\left(\Lambda^{\prime}\right)$ is closed under extensions. By the inductive hypothesis $X^{\prime}$ is a separated monic $\Lambda^{\prime}$-module satisfying the condition $(S G)$, thus $X_{i}$ is strongly Gorenstein-projective for each $i \in Q_{0}$.

By the proof of Lemma 5.7 of [19], we have

$$
\begin{aligned}
\operatorname{Cok}_{i}(X) & =X_{i} / \underset{\beta \in \mathcal{A}(\cdot \rightarrow i)}{\bigoplus} \operatorname{Im} X_{\beta} \\
& \cong\left(X_{i} / \bigoplus_{p \in \mathcal{P}(n \rightarrow i)} \operatorname{Im} X_{p}\right) / \bigoplus_{\substack{\alpha \in Q_{1}^{\prime} \\
e(\alpha)=i}} \operatorname{Im} \widetilde{X_{\alpha}}=\operatorname{Cok}_{i}(\operatorname{Cok} \phi) .
\end{aligned}
$$

Since Cok $\phi$ is a strongly Gorenstein-projective $\Lambda^{\prime}$-module, by the inductive hypothesis we know that $\operatorname{Cok}_{i}(X)=X_{i} / \bigoplus_{\beta \in \mathcal{A}(\rightarrow i)} \operatorname{Im} X_{\beta}$ is a strongly Gorenstein-projective $A$-module. Hence $X$ satisfies the condition $(S G)$.

\section{Applications}

In 8], Bennis, Mahdou and Ouarghi characterized a class of rings over which all modules are strongly Gorenstein-projective. In [21], Mahdou and Tamekkante extended the above idea. They discussed the rings over which every Gorenstein-projective module is strongly Gorenstein-projective. By the main result Theorem 3.2, we can get the following results.

Corollary 4.1. Let $Q$ be a finite acyclic quiver, $A$ a finite-dimensional algebra over a field $k$, and $\Lambda=A Q$. Then $\mathcal{S G P}(\Lambda)=\mathcal{G P}(\Lambda)$ if and only if $\mathcal{S G P}(A)=\mathcal{G P}(A)$.

Proof. Firstly, we prove the necessity of this assertion. For each $M \in \mathcal{G P}(A)$, let $P_{1}(M)$ be the $\Lambda$-module with $P_{1}(M)_{1}=M$ and $P_{1}(M)_{i}=0$ for $i \neq 1$. It is easy to see that $P_{1}(M)$ is a separated monic representation satisfying the condition $(G)$. Then, by Theorem 5.1 of [19], $P_{1}(M) \in \mathcal{G P}(\Lambda)$. Since $\mathcal{S G P}(\Lambda)=\mathcal{G P}(\Lambda)$, we have $P_{1}(M) \in \mathcal{S G P}(\Lambda)$. Hence, by Theorem 3.2, $M=P_{1}(M)_{1} \in \mathcal{S G P}(A)$. That is to say, $\mathcal{S G P}(A)=\mathcal{G P}(A)$.

Conversely, assume that $\mathcal{S G P}(A)=\mathcal{G P}(A)$. Let $X \in \mathcal{G} \mathcal{P}(\Lambda)$. Then by Theorem 5.1 of [19], we have that $X \in \operatorname{smon}(Q, A)$ and both $X_{i}$ and $\operatorname{Cok}_{i}(X)=X_{i} / \bigoplus_{\alpha \in \mathcal{A}(\cdot \rightarrow i)} \operatorname{Im} X_{\alpha}$ 
are Gorenstein-projective $A$-module for each vertex $i \in Q_{0}$. Since $\mathcal{S G P}(A)=\mathcal{G P}(A)$, we have that both $X_{i}$ and $X_{i} / \bigoplus_{\alpha \in \mathcal{A}(\rightarrow \rightarrow)} \operatorname{Im} X_{\alpha}$ are strongly Gorenstein-projective. That is to say, $X$ satisfies the condition $(S G)$. So by Theorem 3.2, we have $X \in \mathcal{S G P}(\Lambda)$. Hence $\mathcal{S G P}(\Lambda)=\mathcal{G P}(\Lambda)$.

For a field $k$ and an integer $t \geq 2$, the algebra $A=k[x] /\left\langle x^{t}\right\rangle$ is self-injective and all the $A$-modules are Gorenstein-projective. So we have

Corollary 4.2. Let $Q$ be a finite acyclic quiver, $A=k[x] /\left\langle x^{t}\right\rangle$ where $k$ is a field and $t \geq 2$ is an integer, and $\Lambda=A Q$. Then each Gorenstein-projective $\Lambda$-module is strongly Gorenstein-projective if and only if $A=k[x] /\left\langle x^{2}\right\rangle$.

Proof. For $A=k[x] /\left\langle x^{2}\right\rangle$ the dual number algebra, Ringel and Zhang have proved that every Gorenstein-projective $\Lambda$-module is strongly Gorenstein-projective via the definition of strongly Gorenstein-projective module in [23].

Conversely, assume that every Gorenstein-projective $\Lambda$-module is strongly. Then, by Corollary 4.1, every Gorenstein-projective $A$-module is strongly too. Since $A$ is selfinjective, every $A$-module is Gorenstein-projective. Then every $A$-module is strongly Gorenstein-projective. Let $S$ be the simple $A$-module, and let $A \rightarrow S$ be the projective cover of $S$, then there is an exact sequence $0 \rightarrow K \rightarrow A \rightarrow S \rightarrow 0$. Since $S$ is a strongly Gorenstein-projective module, by Proposition 2.9 of [7], there is an exact sequence $0 \rightarrow S \rightarrow T \rightarrow S \rightarrow 0$ with $T$ being a projective $A$-module. Then we have a commutative diagram

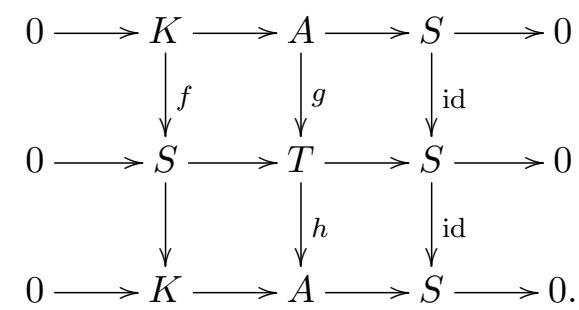

Since $A \rightarrow S$ is right minimal, $h g$ is an isomorphism. So $g$ is a monomorphism. By the snake Lemma, $f$ is a monomorphism too. Hence $f$ is an isomorphism following from the simpleness of $S$. So we have $\operatorname{dim}_{k} A=2$. That is to say, $A=k[x] /\left\langle x^{2}\right\rangle$.

\section{Acknowledgments}

We sincerely thank the referee for very carefully reading the manuscript and many helpful comments and valuable suggestions which helped improving this paper substantially. 


\section{References}

[1] J. Asadollahi, R. Hafezi and M. H. Keshavarz, Minimal injective resolutions and Auslander-Gorenstein property for path algebras, Comm. Algebra 45 (2017), no. 6, $2557-2568$.

[2] M. Auslander and M. Bridger, Stable Module Theory, Memoirs of the American Mathematical Society 94, American Mathematical Society, Providence, R.I., 1969.

[3] M. Auslander and I. Reiten, Cohen-Macaulay and Gorenstein Artin algebras, in: Representation Theory of Finite Groups and Finite-dimensional Algebras (Bielefeld, 1991), 221-245, Progr. Math. 95, Birkhäuser, Basel, 1991.

[4] M. Auslander, I. Reiten and S. O. Smalø, Representation Theory of Artin Algebras, Cambridge Studies in Advanced Mathematics 36, Cambridge University Press, Cambridge, 1995.

[5] L. L. Avramov and A. Martsinkovsky, Absolute, relative, and Tate cohomology of modules of finite Gorenstein dimension, Proc. London Math. Soc. (3) 85 (2002), no. $2,393-440$.

[6] A. Beligiannis, Cohen-Macaulay modules, (co)torsion pairs and virtually Gorenstein algebras, J. Algebra 288 (2005), no. 1, 137-211.

[7] D. Bennis and N. Mahdou, Strongly Gorenstein projective, injective, and flat modules, J. Pure Appl. Algebra 210 (2007), no. 2, 437-445.

[8] D. Bennis, N. Mahdou and K. Ouarghi, Rings over which all modules are strongly Gorenstein projective, Rocky Mountain J. Math. 40 (2010), no. 3, 749-759.

[9] E. Enochs, J. R. García Rozas, L. Oyonarte and S. Park, Noetherian quivers, Quaest. Math. 25 (2002), no. 4, 531-538.

[10] E. E. Enochs and I. Herzog, A homotopy of quiver morphisms with applications to representations, Canad. J. Math. 51 (1999), no. 2, 294-308.

[11] E. E. Enochs and O. M. G. Jenda, On Gorenstein injective modules, Comm. Algebra 21 (1993), no. 10, 3489-3501.

[12] _ Gorenstein injective and projective modules, Math. Z. 220 (1995), no. 4, 611-633.

[13] N. Gao and P. Zhang, Gorenstein derived categories, J. Algebra 323 (2010), no. 7, 2041-2057. 
[14] H. Holm, Gorenstein homological dimensions, J. Pure Appl. Algebra 189 (2004), no. 1-3, 167-193.

[15] W. Hu, X.-H. Luo, B.-L. Xiong and G. Zhou, Gorenstein projective bimodules via monomorphism categories and filtration categories, J. Pure Appl. Algebra 223 (2019), no. $3,1014-1039$.

[16] O. Iyama, K. Kato and J.-I. Miyachi, Recollement of homotopy categories and CohenMacaulay modules, J. K-Theory 8 (2011), no. 3, 507-542.

[17] F. Li and C. Ye, Gorenstein projective modules over a class of generalized matrix algebras and their applications, Algebr. Represent. Theory 18 (2015), no. 3, 693-710.

[18] Z.-W. Li and P. Zhang, A construction of Gorenstein-projective modules, J. Algebra 323 (2010), no. 6, 1802-1812.

[19] X.-H. Luo and P. Zhang, Monic representations and Gorenstein-projective modules, Pacific J. Math. 264 (2013), no. 1, 163-194.

[20] S Separated monic representations I: Gorenstein-projective modules, J. Algebra 479 (2017), 1-34.

[21] N. Mahdou and M. Tamekkante, When every Gorenstein projective (resp. flat) module is strongly Gorenstein projective (resp. flat), Commun. Math. Appl. 1 (2010), no. 1, $15-25$.

[22] C. M. Ringel, Tame Algebras and Integral Quadratic Forms, Lecture Notes in Mathematics 1099, Springer-Verlag, Berlin, 1984.

[23] C. M. Ringel and P. Zhang, Representations of quivers over the algebra of dual numbers, J. Algebra 475 (2017), 327-360.

[24] X. Yang and Z. Liu, Strongly Gorenstein projective, injective and flat modules, J. Algebra 320 (2008), no. 7, 2659-2674.

[25] P. Zhang, Monomorphism categories, cotilting theory, and Gorenstein-projective modules, J. Algebra 339 (2011), 181-202.

[26] _ Gorenstein-projective modules and symmetric recollements, J. Algebra 388 (2013), 65-80.

Tengxia Ju and Xiu-Hua Luo

Department of Mathematics, School of Sciences, Nantong University, Jiangsu 226019,

China

E-mail addresses: jtx@ntu.edu.cn, xiuhualuo@ntu.edu.cn 